\title{
VIEW FROM HERE
}

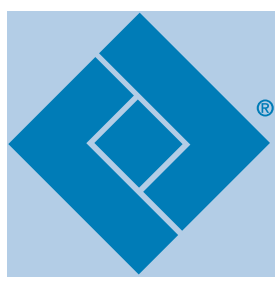

\section{Cost-effectiveness of a WOC Advanced Practice Nurse in the Acute Care and Outpatient Setting}

Jenny A. Medley

\section{ABSTRACT}

Increasing numbers of advanced practice nurses who practice within the WOC specialty are challenged by the need to justify their role by demonstrating clinical and fiscal benefits to the employing agency. This View From Here column describes the steps I took while completing such an analysis for a position for a nurse practitioner with WOC certification in upstate New York.

KEY WORDS: acute care, business proposal, cost-effectiveness, hospital acquired pressure ulcer (PU), outpatient care

\section{Introduction}

A certified WOC nurse (CWOCN) with an advanced practice (AP) degree has much to offer in both the inpatient and outpatient settings. Nevertheless, in the current health care climate, it is often necessary to prove that such a position will provide fiscal as well as clinical benefits to potential employers. I recently completed a graduate program in administration and management, and the capstone project required for this program involved development of a potential solution to a real-life problem currently faced by health care agencies. I chose to develop a business proposal for employment of an AP CWOCN and implementation of a comprehensive wound and ostomy program. I chose this project based on recognition of significant gaps in wound and ostomy care currently found in many agencies. For example, a major challenge in many facilities is the need to reduce or eliminate stage III and stage IV hospital-acquired pressure ulcers (PUs). Their elimination has fiscal as well as clinical impact; as part of the Deficit Reduction Act of 2005, the Centers for Medicare \& Medicaid Services has eliminated payment for these wounds. ${ }^{1}$ An AP CWOCN can also provide outpatient wound services; this is particularly critical in underserved areas where patients are forced to travel long distances to receive specialized wound, ostomy or continence care. Finally, I recognized that an AP CWOCN can provide preoperative and postoperative rehabilitative care for new ostomy patients, thus improving clinical outcomes and health-related quality of life in this underserved population.

\section{Literature Review}

Based on an integrative literature review spanning 2010 to 2012, I identified articles that demonstrated how nurse practitioners can help meet health care needs in areas suffering from physician shortages. ${ }^{2}$ Certified WOC nurses can provide excellence in prevention, health maintenance, therapeutic intervention, and rehabilitative care for persons with gastrointestinal, genitourinary, or integumentary system problems. ${ }^{3}$ Johnson ${ }^{4}$ described the importance of follow-up care for stoma patients, who are at risk for physical, psychological, and social problems. Approximately $20 \%$ to $71 \%$ of persons with ostomies experience complications, the most common being pouch leakage and peristomal skin problems. Williams ${ }^{5}$ stated that peristomal skin problems affect one-third of colostomy patients and two-thirds of urostomy and ileostomy patients. The support and education needed to prevent peristomal skin problems begin with early referral and ongoing access to a CWOCN. ${ }^{5}$

Another area where WOC nurses can exert a clinically relevant impact is prevention and management of PUs and other types of skin damage. Health care facilities

\footnotetext{
- Jenny A. Medley, RN, MSN, Samaritan Medical Center, Walden University, 23929 Pheasant Run, Watertown, NY 13601 (jmedley@ twcny.rr.com).

The author declares no conflict of interest.

This is an open-access article distributed under the terms of the Creative Commons Attribution-NonCommercial-NoDerivitives 3.0 License, where it is permissible to download and share the work provided it is properly cited. The work cannot be changed in any way or used commercially.

Correspondence: Jenny A. Medley, RN, MSN, Samaritan Medical Center, Walden University, 23929 Pheasant Run, Watertown, NY 13601 (jmedley@twcny.rr.com).
}

DOI: 10.1097/WON.0000000000000039 
report higher costs of care and significantly prolonged length of stay for patients with PUs; average lengths of stay for these patients can be as high as 3 times the length of stay for patients without a PU. ${ }^{6}$ A nurse practitioner certified in WOC nursing can develop and manage a comprehensive program for PU prevention and management and contribute significantly to improved outcomes for all patients with skin and wound care issues, including incontinence-associated dermatitis and fistulas. ${ }^{6}$ Staff education is a key element of a comprehensive wound and ostomy care program, and AP CWOCNs are well prepared to provide this education.

\section{Description of Health Care Needs}

Samaritan Medical Center in Watertown, New York, is located in the northwest corner of New York State in Jefferson County. The city of Watertown is the major population, commercial and industrial center as well as county seat for Jefferson County. It has a population of 117,162.7 According to the Jefferson County Community Health Assessment 2010-2013, morbidity and mortality associated with diabetes mellitus is a priority health concern. Diabetes prevalence in adults in Jefferson County is $10.2 \%$, which is higher than the national average of $7.5 \%{ }^{8}$ This translates to 11,950 persons with diabetes living in Jefferson county. Based on these statistics, 15\% are expected to develop diabetic foot ulcers. In addition, $0.55 \%$ of the primary population is likely to develop venous ulcers, and $0.85 \%$ of the primary population is at risk for development of PU. ${ }^{8}$ Currently, the closest wound care centers are located 70 miles south and 59 miles northeast of Watertown.

Samaritan Medical Center is a 294-bed nonprofit medical center within a 3-building campus, including a 272-bed long-term care facility and a new 288-bed long-term care and assisted living combined facility. The medical center offers a spectrum of inpatient and outpatient medical and surgical services. In 2013, a urologist was hired who will perform robotic assisted urologic cancer and reconstructive procedures using a DaVinci system (Intuitive Surgical, Sunnyvale, California). The addition of these surgical services has increased the number of ostomy patients and the need for expertise in ostomy care. ${ }^{9}$

The mission and vision statements of Samaritan Medical Center state that the facility will provide "high quality, comprehensive, safe, and compassionate health care services to meet the needs of our civilian and military community," and its vision statement identifies the need to provide specialty care services for community residents. ${ }^{10}$ These statements clearly reflect a commitment to meeting the health care needs of the community. However, the closest wound care centers are located some distance away. This creates a gap in care when one considers the large diabetic population in Jefferson County. In addition, hiring a urologist in 2013 increased the need for an ostomy nurse specialist and for staff education in this area of care.

\section{Market Analysis}

My project focused on creation of a WOC NP position within the Samaritan Medical Center. When a market analysis was performed on inpatient figures to try to justify such a position, the inpatient totals amounted to an average of 356 patients per year. This number was generated from the average estimated urology surgeries performed per year (25), plus the average number of patients seen within the organization with all types of ostomy (163), plus the number of inpatient wound consults completed per year (150), and the average wound care services provided by same-day surgery (18). The sum of these equals 356 patients resulting in approximately 1 patient per day in a calendar year (356 patients/352 days per year $=1.01$ ).

Evaluation of outpatient services identified a greater demand for AP CWOCN services. As noted earlier, approximately 11,950 persons in Jefferson county are estimated to suffer from diabetes mellitus, and 15\% are anticipated to develop diabetic foot ulcers, resulting in 1793 patients. In addition, $0.55 \%$ of the county's population $(117,162 \times$ $0.0055)$ is anticipated to develop venous leg ulcers, resulting in 644 patients, and 1031 residents are expected to develop a PU (representing $0.88 \%$ of the county's current population or $117,162 \times 0.0088)$. The combined potential outpatients resulted in 3468 potential patients requiring WOC nursing services $(1793+644+1031)$.

\section{Strength, Weakness, Opportunity, Threat Analysis}

A Strength, Weakness, Opportunity, Threat (SWOT) analysis is a strategic planning activity used to generate and analyze data and ideas in order to identify strengths, weaknesses, opportunities, and threats associated with a given program or organization. ${ }^{11}$ Identified strengths associated with creating an AP CWOCN-run program included the comparatively high percentage of the population with diabetes mellitus, the lack of local competition for an outpatient clinic (based on the fact that the closest wound center is 59 miles away), and the development of a urologic services with a robotic assisted laparoscopic surgical program. Weaknesses included decreased hospital admissions in 2013, lack of a WOC certified NP, absence of an established outpatient wound clinic in the area, and the low numbers of inpatients likely to require wound and ostomy services. Opportunities included the lack of an established wound clinic, a paucity of nurses certified in wound care, and the need for a comprehensive program to eliminate or minimize hospital-acquired pressures ulcers (HAPUs). Threats include the potential 
competition provided by the wound centers in adjacent communities and the reduction in inpatient revenue associated with hospital-acquired stage III and stage IV PUs, which are not reimbursable by the Centers for Medicare \& Medicaid Services. Based on this SWOT analysis, I concluded that Samaritan Medical Center should invest in development of outpatient wound services, an aggressive program for prevention of hospital-acquired stage III and stage IV PUs, further development of a urologic service with robotic assisted laparoscopic surgery, and investment in nurses certified in wound care.

\section{Financial Data}

I began by trying to justify the cost of the AP WOC nurse position, using data gathered concerning inpatient needs. I assumed that a visit fee of $\$ 125$ would be charged for a typical inpatient visit and that, on average, contractual agreements would result in a 50\% reduction in the payment to the agency. Using an average number of 356 inpatient visits calculated earlier, the gross revenue was estimated to be $\$ 44,500$. A contractual adjustment of $50 \%$ resulted in a net revenue of $\$ 22,250$. I then compared potential revenue to the cost of a full-time AP WOC nurse, using an average nurse practitioner salary of $\$ 79,997$ per year and nonsalary expenses for supplies, travel, education, marketing, and food of $\$ 10,000$ per year; this resulted in a total cost figure of $\$ 89,997$. When I compared the projected net revenue of $\$ 22,250$ to the estimated cost of an AP WOC nurse, the facility projected a loss of $\$ 67,747$. Based on these estimated costs, I was unable to justify employment of a full-time AP WOC nurse for the medical center at this time. However, this analysis did not exclude establishment of a part-time position or development of a "combination" inpatient/outpatient position.

It should be noted that the payer mix must be considered when calculating potential revenue, since Medicare is the primary payer for many agencies and Medicare payments are based on diagnoses as opposed to services provided. This payment system means that an agency for which Medicare is the primary source would realize little additional revenue from the addition of WOC nurse services provided by a hospital employee. In this situation, the WOC nurse must focus on ways in which her or his services provide cost savings (eg, by reducing costly HAPUs and assuring appropriate use of costly therapies such as support services and negative pressure wound therapy) in order to justify the cost of her or his position. Another option is for the WOC AP nurse to provide services as an independent provider as opposed to a hospital employee. In this case, the AP WOC nurse would obtain hospital privileges and bill Medicare directly for services rendered.

In contrast, analysis of costs related to providing outpatient services proved more promising. Given the potential number of outpatients (3468) multiplied by an average visit charge ( $\$ 125$ ) resulted in a gross revenue of $\$ 433,500$. Calculation of the estimated net revenue for an AP WOC nurse required subtraction of a contractual adjustment $(50 \%)$, yielding a net revenue of $\$ 216,750$. I then subtracted the salary and nonsalary expenses (including a 3\% yearly increase to $\$ 92,697$ ), which yielded a potential net income of $\$ 124,053$. These numbers can be described as conservative because they are based on only 1 visit per patient. Given the nature of the services required by patients with chronic wounds or ostomies, it is more likely that each patient would require multiple visits and the increased number of actual visits per patient is projected to easily offset any reduction in the total number of wound patients referred to the center.

\section{Break Even Analysis}

A break even analysis was performed to determine the number of visits needed per day to justify the cost of a WOC NP position. The total expense, $\$ 89,997$, was divided by the average payment after contractual allowance (\$63), resulting in 1429 visits to break even. Assuming an average

\section{BOX 1.}

\section{Future Suggestions and Ideas}

Based on the analysis described in this article, the following conclusions and alternative strategies were presented to nursing management and the senior management team for consideration. Senior management was receptive to these ideas and is considering developing a wound care program for the entire system.

1. Hire an AP CWOCN who sees inpatients and has a dual role of assisting surgeons during robotic surgery as first assistant.

2. Allow multiple RNs to achieve WOC certification and see inpatients as before, forgoing the cost of the nurse practitioner.

3. Implement an outpatient wound clinic with an NP and wound care team including other wound certified nurses to see a large range of wound and ostomy patients. The outpatient clinic can be associated with the infusion unit, diabetic or other established clinic on the SMC campus. The outpatient WOC NP could also provide inpatient coverage.

4. Explore an agreement/affiliation with other wound clinics.

5. Make no change, but risk losing a source of revenue for the organization as well as a specialty service that could be offered to the community.

6. Consider hiring a WOC NP to provide services throughout the multicampus community with goal of preventing costly HAPUs in acute and long-term care. 
work week of 5 eight-hour days over a period or 50 weeks per year (250 working days), the AP WOC nurse would need to see 5.7 patients per day to break even (1429 visits/250 working days). This estimate provided fiscal justification for a full-time nurse practitioner in the outpatient setting. In addition, the break even analysis is well below the projected number of visits per day (13.87) occurring in the outpatient setting (this figure is based on a calculation of 3468 visits divided by 250 days). These data suggest that the best approach for this medical center at this time is to consider establishment of a WOC AP position that covers both the inpatient and outpatient settings. If we are able to establish such a position, it will be important to maintain accurate data regarding visit numbers and revenue, as we anticipate that the need for WOC nurse services will continue to grow. Box 1 summarizes elements of the business proposal presented to nursing management and senior team members at the Samaritan Medical Center.

\section{Conclusions}

My analysis provides support for multiple benefits in hiring an AP WOC nurse, including improved clinical outcomes, prevention of HAPUs, and the education of patients, families, and employees in wound and ostomy care. Fiscal considerations for the hiring agency should include not only salary and benefits, but also community need, payer mix, potential for growth, and potential competition for services in the area.

\section{KEY POINTS}

WOC nurses must be able to prove the fiscal as well as clinical benefits of their services.

WOC nurses produce excellent patient outcomes, reduce costs, and increase patient satisfaction by acting as direct caregivers, educators, consultants, and researchers in a variety of settings. In the inpatient setting, WOC nurses can implement programs to minimize or eliminate HAPUs and can optimize outcomes and reduce readmission rates for new ostomy patients.

In settings where there is insufficient patient census to justify a WOC nurse position, AP WOC nurses can provide additional services, such as assistance with robotic surgery.

\section{References}

1. Centers for Medicare \& Medicaid Services. Medicaid integrity program report to Congress. http://www.cms.gov/Regulationsand-Guidance/Legislation/DefecitReductionAct/repcongress. html. Published 2012.

2. Lucero R, Rauch L, Berkowitz B. Nurse practitioner workforce: a substantial supply of primary care providers. Nurs Econ. 2012;30(5):268-294.

3. Lawrence K, Catanzaro J, Eddins C, Jacobson T, Slachta P. Scope and standards for wound, ostomy and continence specialty practice nursing: a white paper from the WOCN society. $J$ Wound Ostomy Continence Nurs. 2012;39(5):481-487.

4. Johnson T. Follow-up care of stoma patients: a systematic literature review. Gastrointest Nurs. 2012;10(9):30-36.

5. Williams J. Healthy peristomal skin is essential. Br J Nurs. 2011;20(22):1408.

6. Jankowski I. Matching patient safety goals to the nursing specialty: using wound, ostomy, continence nursing services. $J$ Nurs Adm. 2010;40(1):26-31.

7. Jefferson County Public Health Service. 2010-2013 community health assessment. http://www.co.jefferson.ny.us/Modules/ ShowDocumnet.aspx?documentid = 2689. Published 2010.

8. National Institutes of Health. Human skin wounds: a major and snowballing threat to public health and the economy. Wound Repair Regen. 2009;17(6):763-771. http://www.ncbi. nlm.nih.gov/pubmed/19903300.

9. Zender J, Thell C. Developing a successful robotic surgery program in a rural hospital. AORN J. 2010;92(1):72-86. doi: 10.1016/j.aorn.2009.10.024.

10. Samaritan Medical Center. Our story and mission. http://www .samaritanhealth.com. Published 2013.

11. DeSilets L, Dickerson P. SWOT is useful in your tool kit. J Contin Educ Nurs. 2008;39(5):196-197. 